\title{
Percepção dos enfermeiros nefrologistas acerca do processo de ensino aprendizagem em unidades de hemodiálise
}

\author{
Perception of nephrologist nurses about the teaching learning process in hemodialysis units \\ Percepción de enfermeras nefrológicas sobre el proceso de enseñanza aprendizaje en unidades de hemodiálisis
}

\begin{abstract}
RESUMO
Objetivo: Verificar a percepção do processo de ensino aprendizagem em unidades de hemodiálise dos enfermeiros nefrologistas de todas as regiões brasileiras através de um diagnóstico situacional. Método: A pesquisa foi realizada através da ferramenta de formulários do Google drive, no período de novembro de 2019 a fevereiro de 2020. Participaram da pesquisa 71 enfermeiros nefrologistas de todas as regiões do Brasil. Resultados: Os principais achados foram relacionados ao tempo de atuação dos enfermeiros na nefrologia, método e ferramenta utilizados em treinamentos nas unidades de diálise, média de tempo para o treinamento em uma nova tecnologia, a estratégia favorita dos enfermeiros, conhecimento sobre tecnologia educacional e o interesse em ter uma ferramenta que auxiliasse na educação permanente dos enfermeiros na hemodiálise. Conclusão: Com base nas respostas obtidas nesse diagnóstico situacional fica clara a necessidade de ferramentas que possam auxiliar no processo de ensino aprendizagem em unidades de hemodiálise, fazendo-se necessário mais pesquisas sobre o tema a fim de desenhar novas tecnologias para auxiliar na informatização dos enfermeiros nefrologistas que atuam em unidades de hemodiálise.
\end{abstract}

DESCRITORES: Diagnóstico da Situação de Saúde;Enfermagem em Nefrologia; Hemodiálise; Tecnologia Educacional.

\section{ABSTRACT}

Objective: To verify the perception of the teaching-learning process in hemodialysis units of nephrology nurses from all Brazilian regions through a situational diagnosis. Method: The survey was conducted using the Google drive forms tool, from November 2019 to February 2020. 71 nephrologist nurses from all regions of Brazil participated in the survey. Results: The main findings were related to the length of experience of nurses in nephrology, method and tool used in training in dialysis units, average time for training in a new technology, the nurses' favorite strategy, knowledge about educational technology and the interest in having a tool that would help in the continuing education of nurses in hemodialysis. Conclusion: Based on the answers obtained in this situational diagnosis, the need for tools that can assist in the teaching-learning process in hemodialysis units is clear, making more research on the subject necessary in order to design new technologies to assist in the teaching process for nephrology nurses working in hemodialysis units.

DESCRIPTORS: Diagnosis of Health Situation; Nephrology Nursing; Hemodialysis; Educational Technology.

\section{RESUMEN}

Objetivo: Verificar la percepción del proceso de enseñanza-aprendizaje en unidades de hemodiálisis de enfermeras de nefrología de todas las regiones brasileñas a través de un diagnóstico situacional. Método: La encuesta se realizó utilizando la herramienta Google Drive Forms, del de noviembre de 2019 al febrero de 2020. Participaron de la encuesta 71 enfermeras de nefrología de todas las regiones de Brasil. Resultados: Los principales hallazgos se relacionaron con la antigüedad de los enfermeros en nefrología, el método (s) y herramienta (s) utilizados en la formación en las unidades de diálisis, el tiempo medio de formación en una nueva tecnología, el método de formación habitual de servicio y un favorito de las enfermeras, conocimiento sobre tecnología educativa e interés en contar con una herramienta que ayude en la formación continua de enfermeras en hemodiálisis. Conclusión: A partir de las respuestas obtenidas en este diagnóstico situacional, es clara la necesidad de herramientas que puedan ayudar en el proceso de enseñanza-aprendizaje en las unidades de hemodiálisis, haciendo necesaria más investigación sobre el tema para diseñar nuevas tecnologías que ayuden en el proceso de enseñanza. para enfermeros nefrólogos que trabajan en unidades de hemodiálisis.

DESCRIPTORES: Diagnóstico de la Situación de Salud; Enfermería en Nefrología; Hemodiálisis; Tecnología Educativa.

RECEBIDO EM: 12/10/2021 APROVADO EM : 15/11/2021 


\section{Claudia Maria Marinho de Almeida Franco}

Mestre em Tecnologia e Inovação em Enfermagem pela Universidade de Fortaleza (UNIFOR)

ORCID: 0000-0003-1926-7592

\section{Isabela Melo Bomfim}

Mestre e Doutora em Enfermagem Clinico Cirúrgica pela Universidade Federal do Ceará- UFC. Docente em graduação em Enfermagem e do Mestrado Profissional Tecnologia e Inovação em Enfermagem da Universidade de Fortaleza - UNIFOR. CE-Brasil.

ORCID: 0000-0002-0056-862X

\section{Rita Mônica Borges Studart}

Doutora em Enfermagem pela Universidade Federal do Ceará. Especialista em Nefrologia e Bloco cirúrgico. Docente do Mestrado Profissional Tecnologia e Inovação em Enfermagem. CE-Brasil

ORCID: 0000-0002-5862-5244

\section{Francisca Elisângela Teixeira Lima}

Doutora em Enfermagem. Docente da Universidade Federal do Ceará-UFC.

ORCID: 0000-0002-7543-6947

\section{Gleison Resende Sousa}

Mestre em Tecnologia e Inovação em Enfermagem, Especialista em Urgência e Emergência. CE-Brasil.

ORCID: 0000-0001-5805-9281

\section{Kalyni Silvino Serra}

Graduanda em enfermagem pela Universidade de Fortaleza - UNIFOR.

ORCID: 0000-0002-0542-4692

\section{INTRODUÇÃO}

$\mathbf{H}$ emodiálise é o método de tratamento renal substitutivo mais utilizado no Brasil e que segundo Portaria 1675 de 7 de junho de 2018 do Ministério da Saúde do Brasil exige enfermeiros especialistas em nefrologia. Devido as particularidades inerentes ao tratamento de hemodiálise a educação permanente de enfermeiros é imprescindível para a qualidade da assistência prestada aos pacientes ${ }^{1}$.

Dito isso, a fim de conhecer a percepção dos enfermeiros nefrologistas sobre o melhor método de ensino aprendizagem para enfermeiros em unidades de hemodiálise foi realizado um diagnóstico situacional sobre o tema. O diagnóstico situacional auxilia no conhecimento dos problemas e necessidades, através da utilização de ferramentas de pesquisas, que podem ser questionários, entrevistas estruturadas ou semiestruturadas, entre outras ${ }^{2}$.

Questionário é um instrumento para o levantamento de dados por amostragem, é um método que auxilia na coleta de informações das pessoas acerca de suas ideias.
Hemodiálise é o

método de tratamento

renal substitutivo mais

utilizado no Brasil e

que segundo Portaria

1675 de 7 de junho de

2018 do Ministério

da Saúde do Brasil

exige enfermeiros

especialistas em

nefrologia
Assim, a construção de um bom questionário, ou seja, de um instrumento eficaz de avaliação, depende do conhecimento de técnicas para elaboração das perguntas e principalmente, das experiências do pesquisador ${ }^{3}$.

Planejamento, identificação das fontes e a coleta dos dados, além da análise e apresentação dos resultados são a base para pesquisadores e profissionais. Atualmente na área da saúde há inúmeros questionários e escalas que são utilizados para delinear o desenho da pesquisa. O questionário, deve ser confiável, as perguntas devem ser pertinentes e com condições para serem medidas 4.

As mudanças nos processos de ensino, com utilização de metodologias ativas e foco na andragogia, saindo do ensino tradicional e buscando cada vez mais atender as necessidades do aluno, vem trazendo melhores e maiores resultados 5 .

Neste contexto, foram formuladas questões com o intuito de conhecer: o tempo de atuação dos enfermeiros na nefrologia, método(s) e ferramenta(s) utilizados em treinamentos nas unidades de diálise, média 


\section{artigo}

Franco, C. M. M. A., Bomfim, I. M., Stuardt, R. M. B., Lima, F. E. T., Sousa, G. R., Serra, K. S.

Percepção dos enfermeiros nefrologistas acerca do processo de ensino aprendizagem em unidades de hemodiálise

de tempo para o treinamento em uma nova tecnologia, o método de treinamento habitual do serviço e o favorito dos enfermeiros, conhecimento sobre tecnologia educacional e o interesse em ter uma ferramenta que auxiliasse na educação permanente dos enfermeiros na hemodiálise.

Objetivou-se verificar a percepção do processo de ensino aprendizagem em unidades de hemodiálise dos enfermeiros nefrologistas de todas as regiões brasileiras através de um diagnóstico situacional.

\section{MÉTODO}

Trata-se de uma pesquisa descritiva, a qual tem a finalidade de conhecer e interpretar a realidade sem nela interferir para modificá-la 6.

Para o alcance do objetivo proposto pelo estudo, foi realizado um diagnóstico situacional, através de questionário, distribuído através da ferramenta Google Form, a fim de ter uma avaliação mais clara da percepção dos enfermeiros nefrologistas sobre o processo de aprendizagem para a realização de suas atividades.

Inicialmente o questionário foi disponibilizado como um teste piloto para três enfermeiros nefrologistas, que também atuam na docência, para a avaliação das questões quanto a clareza, relevância teórica e pertinência prática. Vale salientar que os três enfermeiros que participaram do teste piloto não participaram da amostra do estudo.

Após serem analisadas pela pesquisadora as sugestões foram acatadas e os questionários enviados para os participantes da pesquisa através da ferramenta Google Form, seguindo o método "bola de neve".

Esse método, é uma forma de amostragem não probabilística que utiliza cadeias de referência, sendo útil para pesquisar grupos difíceis de serem acessados ou estudados, bem como quando não existe precisão sobre sua quantidade 7 .

O questionário foi formatado e distribuído através da ferramenta virtual "google form" aos enfermeiros nefrologistas de todas as regiões brasileiras que aceitaram participar da pesquisa. Os critérios de inclusão foram: enfermeiros nefrologistas que atuem em serviços de hemodiálise de todas as regiões brasileiras e de exclusão: enfermeiros sem especialização em nefrologia.

$\mathrm{O}$ período de coleta dos dados se deu durante 79 dias, no período do dia 24 de novembro de 2019 a 11 de fevereiro de 2020.

Todos os dados registrados pelos participantes da pesquisa são confidenciais respeitando a Resolução 466/12, que regulamenta as normas e diretrizes para a pesquisas envolvendo seres humanos.

O presente estudo foi realizado conforme aprovação do Comitê de Ética em Pesquisa (CEP) com número CAAE 18584619.7.0000.5052 e parecer número: 3.644 .994 .

\section{RESULTADOS}

Participaram do estudo 71 enfermeiros de todas as regiões do país e 67 respostas válidas. Dois enfermeiros da região norte, 30

\begin{tabular}{|c|c|}
\hline Regiões & $\begin{array}{c}\text { No Enfermeiros }^{\circ} \\
\text { Por Região }\end{array}$ \\
\hline Norte & 2 \\
\hline Nordeste & 30 \\
\hline Centro Oeste & 5 \\
\hline Sudeste & 22 \\
\hline Sul & 8 \\
\hline TOTAL & 67 \\
\hline
\end{tabular}

enfermeiros da região nordeste, cinco enfermeiros da região centro-oeste, 22 enfermeiros da região sudeste e oito enfermeiros da região sul), após a leitura e aceitação de participação conforme TCLE, constante no cabeçalho do instrumento enviado através da ferramenta Google Forms, conforme demonstrado na tabela 1 .

Tabela 2 - Tempo médio para treinamento de um enfermeiro sem experiência a manusear uma máquina de hemodiálise, Fortaleza-CE,2020

\section{Tempo Médio Para Treinamento De Um Enfermeiro Sem Experi- ência A Manusear Uma Máquina De Hemodiálise}

Não Lembro

$\%$

$22,90 \%$

De Uma A Três Horas De Treinamento

$0 \%$

De Quatro A Seis Horas De Treinamento

$2,80 \%$

Mais De Seis Horas De Treinamento $28,60 \%$

Não Contrata Enfermeiros Sem Experiência Na Área

$45,70 \%$

TOTAL

$100 \%$
Tabela 3 - Método de treinamento mais utilizado nas unidades de diálise pesquisadas, Fortaleza -CE, 2020

\section{Método De Treinamento Mais Utilizado Nas} Unidades De Diálise Pesquisadas

POPs (Procedimento Operacional Padrão)

EAD (Ensino a distância - E-Learning)

Vídeos Educativos

Aulas expositivas

Treinamento específicos in loco

Apostilas

Fonte: Elaborado pelo autor,2020
Total De Respostas 62 18 28 38 2 2
$\%$ $88,6 \%$ $25,7 \%$ $40,00 \%$ $54,30 \%$ $2,80 \%$ $2,80 \%$ 
Tabela 4 - Gostaria de ter uma ferramenta que ajude no direcionamento dos treinamentos?,Fortaleza-CE,2020

$\begin{array}{lcc}\begin{array}{l}\text { Gostaria De Ter Uma Ferramenta Que Ajude } \\ \text { No Direcionamento Dos Treinamentos? }\end{array} & \text { Sim } & \text { Não } \\ \begin{array}{l}\text { Respostas Dos Enfermeiros } \\ \text { TOTAL }\end{array} & 98,60 \% & 1,40 \% \\ \text { Fonte: Elaborado pelo autor,2020 } & & 100 \% \\ \end{array}$

Tabela 5- Tecnologia considerada mais eficiente para o treinamento segundo a pesquisa, Fortaleza - CE,2020

\begin{tabular}{lcc}
\hline $\begin{array}{c}\text { Tecnologia Consideradas Mais Eficientes } \\
\text { Para Treinamento Segundo Os Enfermeiros }\end{array}$ & Total De Respostas & $\%$ \\
POPs (Procedimento Operacional Padrão) & 24 & $34,30 \%$ \\
Cartilhas Educativas & 14 & $20 \%$ \\
EAD (Ensino a distância - E-Learning) & 42 & $34,3 \%$ \\
Vídeos Educativos & 37 & $52,90 \%$ \\
Aulas expositivas & 38 & $54,30 \%$ \\
Treinamento Prático & 1 & $1,40 \%$ \\
Programa trainee & 1 & $1,40 \%$ \\
Fonte: Elaborado pelo autor,2020 & &
\end{tabular}

Tabela 6 - Principais complicadores para a realização de treinamentos na visão dos enfermeiros nefrologista, Fortaleza-CE,2020

$\begin{array}{lc}\begin{array}{lc}\text { Principais Complicadores Para A Realização De Treinamentos Na } \\ \text { Visão Dos Enfermeiros Nefrologistas }\end{array} & \text { \% De Respostas } \\ \text { Investimento econômico para treinamentos } & 25,70 \% \\ \text { Tempo } & 42,90 \% \\ \text { Espaço } & 1,43 \% \\ \text { Motivação da equipe } & 27,10 \% \\ \text { Nenhum } & 0 \% \\ \text { Treinamento no horário de trabalho } & 1,43 \% \\ \text { Donos de clínicas comprarem a ideia } & 1,44 \% \\ \text { TOTAL } & 100,00 \% \\ \text { Fonte: Elaborado pelo autor,202o } & \end{array}$

Conforme os dados registrados tivemos uma predominância maior de respostas de enfermeiros das regiões Nordeste (30) e Sudeste (22) do Brasil. Em resposta à pergunta sobre quanto tempo em média de horas necessitariam para treinar um enfermeiro sem experiência a manusear uma máqui- na de hemodiálise, 28,6\% dos enfermeiros responderam levar mais de 6 horas para o desempenho dessa atividade, se somarmos os 2,8\% que levam 4 a 6 horas para treinar temos $31,4 \%$, O que representa um percentual importante quando falamos de uma atividade considerada básica na formação de profissionais em hemodiálise, conforme demonstrado na tabela 2 .

Ao avaliarmos os métodos de ensino mais utilizados atualmente pelos enfermeiros, a pesquisa nos mostra conforme tabela 3, que os mesmos são: Os POPs, as aulas expositivas e os vídeos educativos, com $88,6 \%, 54,3 \%$ e $40 \%$ respectivamente. O percentual citado se deu pelos enfermeiros terem realizado pelo menos 2 opções de escolha entre perguntas fechadas e aberta no questionário.

Quando perguntados sobre a satisfação com os métodos de treinamento que já utilizam em suas unidades no seu dia a dia, a maioria dos participantes, 68,6\% dizem estarem satisfeitos com os métodos que já utilizam, dando nota maior que 6,0 a sua satisfação.

Apesar de mais de 60\% dos enfermeiros se mostrarem satisfeitos com os métodos atuais de treinamentos em seus serviços de diálise, 98,6\% gostariam de ter uma ferramenta que ajudasse no direcionamento dos treinamentos para diminuir o tempo e aumentar a eficácia dos mesmos, sendo os vídeos educativos, aulas expositivas, POPs e educação a distância os métodos mais desejados para auxiliar nesses treinamentos, conforme tabela 4 .

Quando perguntados sobre quais tecnologias são consideradas como as mais eficientes os participantes da pesquisa responderam que as aulas expositivas e os vídeos educativos, com $54,3 \%$ e $52,9 \%$ respectivamente, são os mais eficientes. A pergunta permitia duas respostas entre respostas fechadas e abertas (Tabela 5).

Ao avaliarmos os principais complicadores para a realização dos treinamentos dos enfermeiros nas unidades de hemodiálise, o tempo (42,9\%), a motivação da equipe $(27,1 \%)$ e o investimento financeiro para treinamentos pelas unidades de diálise $(25,7 \%)$ foram apontados, pelos participantes da pesquisa, como os principais complicadores (tabela 6).

\section{DISCUSSÃO}

A pesquisa mostra que a percepção dos enfermeiros nefrologistas acerca do proces- 


\section{artigo}

Franco, C. M. M. A., Bomfim, I. M., Stuardt, R. M. B., Lima, F. E. T., Sousa, G. R., Serra, K. S.

Percepção dos enfermeiros nefrologistas acerca do processo de ensino aprendizagem em unidades de hemodiálise

so de ensino e aprendizagem na hemodiálise é positiva.

Para Ceccim 8 a Educação Permanente em Saúde pode corresponder à Educação em Serviço, quando esta coloca a pertinência dos conteúdos, instrumentos e recursos para a formação técnica submetida a um projeto de mudanças institucionais ou de mudança da orientação política das ações prestadas em dado tempo e lugar.

Sendo assim, conforme os dados extraídos das respostas de 67 enfermeiros nefrologistas de todas as regiões brasileiras, 48 enfermeiros disseram ter um grau de satisfação entre 6-10 em uma escala de 0-10. Mesmo satisfeitos 98,6\% desses enfermeiros desejam uma ferramenta para ajuda-los a direcionar a educação permanente das unidades de diálise.

Ao tomar como objeto de transformação e de investigação o processo de trabalho, a EP não procura transformar todos os problemas em problemas de capacitação, busca as lacunas de conhecimento e as atitudes que são parte da estrutura expli- cativa dos problemas identificados na vida cotidiana dos serviços 9

No caso, os POPs ainda são as ferramentas bastante utilizadas hoje pelos enfermeiros para a promoção do ensino na hemodiálise, seguido de aulas expositivas. Alguns enfermeiros acreditam que programas de trainee com ferramentas em EAD e vídeos educativos também podem ser úteis no processo de ensino.

Quando questionamos sobre as principais dificuldades para a manutenção de um programa de educação permanente em uma unidade de hemodiálise, vários motivos foram apontados pelos participantes da pesquisa, entre eles tivemos o tempo para estudo com $42,9 \%$ das respostas, seguindo da motivação da equipe para o estudo com $27,1 \%$ e investimento financeiro com $25,7 \%$.

Como limitação do estudo tivemos a dificuldade de participação dos enfermeiros na resposta do questionário, apesar da disponibilização on-line. Segundo o último censo da SBN (Sociedade Brasileira de Nefrologia) de 2018 que o Brasil possui cerca de 786 centros de diálise e se pensarmos que cada centro possui no mínimo 2 enfermeiros, teríamos 1572 enfermeiros nefrologistas, sendo os 71 participantes da pesquisa apenas $4,52 \%$ desses.

\section{CONCLUSÃO}

Com base nas respostas obtidas através desse diagnóstico situacional fica clara a necessidade de ferramentas que possam auxiliar no processo de ensino aprendizagem em unidades de hemodiálise, a fim de minimizar o tempo gasto no processo de obtenção de conhecimento e aumentar a qualidade do conhecimento adquirido para uma prestação de atendimento de maior qualidade ao paciente renal crônico em programa de hemodiálise.

Faz-se necessário mais pesquisas sobre $\mathrm{o}$ tema a fim de desenhar novas tecnologias para auxiliar no processo de ensino para os enfermeiros nefrologistas que atuam em unidades de hemodiálise.

\section{REFERÊNCIAS}

1. Brasil. Portaria $n^{\circ} 1.675$, de 7 de junho de 2018. Altera a Portaria de Consolidação n 3/GM/MS, de 28 de setembro de 2017, e a Portaria de Consolidação $n^{\circ}$ 6/GM/MS, de 28 de setembro de 2017, para dispor sobre os critérios para a organização, funcionamento e financiamento do cuidado da pessoa com Doença Renal Crônica - DRC no âmbito do Sistema Único de Saúde - SUS. 2018;

2. Bauli J D, Matsuda L M. Diagnóstico situacional do serviço de enfermagem de hospital de ensino sob a ótica dos profissionais de nível médio. Revista de Administração em Saúde. 2009;11(43).

3. Chagas A T R. O Questionário na pesquisa científica. Administração on Line. 2000;1

4. Cooper D, Schindler P. Métodos de pesquisa empresarial. Monte McGraw. 2003;
5. Draganov P, Sanna M C. Avaliação das competências dos professores de enfermagem para facilitar a aprendizagem de adultos. Cogitare Enfermagem. 2015;20(3):556-564.

6. Churchill G A. Marketing research: methodological foundations. Chicago: The Dryden Press. 1987;

7. Vinuto J A. A amostragem em bola de neve na pesquisa qualitativa: um debate em aberto. Temáticas. 2014;22(44):203-220.

8. Ceccim R B. Educação Permanente em Saúde: desafio ambicioso e necessário. Interface (Botucatu), Botucatu, 2005; 9(16):161-168.

9. Batista K B C, Gonçalves O S J. Formação dos profissionais de saúde para o SUS: significado e cuidado. Saude soc. 2011; 20(4): 884-899. 